\title{
Phenyliodine(III) Diacetate-Promoted Synthesis of Benzimidazoles
}

\section{Gategory}

Synthesis of

Heterocycles

Key words

arylbenzamidines

phenyliodine(III) diacetate

benzimidazoles

C-H imidation
Significance: Reported is the synthesis of 2-substituted benzimidazoles by the reaction of $\mathrm{N}$-arylamidines with phenyliodine(III) diacetate under mild conditions via an intramolecular oxidative imidation process. The $\mathrm{C}-\mathrm{H}$ activation reaction is proposed to proceed by the formation of free radical intermediates which was partially supported by a free radical inhibition experiment.
Comment: Compounds containing the benzimidazole moiety are reported to possess a number of interesting biological activities (K. Vijaykumar,

A. J. Ahemed J. Chem. Pharm. Res. 2010, 2, 215). Several syntheses of similar 2-substituted benzimidazoles have been reported involving an intramolecular Cu-catalyzed $\mathrm{N}$-arylation (C. Chen et al. J. Org. Chem. 2011, 76, 716). In comparison, the present synthesis occurs under metal-free, mild conditions. However, the reaction suffers from poor regioselectivity for meta-substituted substrates, leading to mixture of isomers. 